\title{
CONSTRUCTION OF GROUP ACTIONS ON FOUR-MANIFOLDS
}

\author{
ALLAN L. EDMONDS
}

\begin{abstract}
It is shown that any cyclic group of odd prime order acts on any closed, simply connected topological 4-manifold, inducing the identity on integral homology. The action is locally linear except perhaps at one isolated fixed point. In the case of primes greater than three a more careful argument is used to show that the action can be constructed to be locally linear.
\end{abstract}

Introduction. In this paper we shall show that every closed, simply connected topological 4-manifold $M^{4}$ admits an action of any cyclic group $\mathbf{Z}_{p}$ of odd prime order $p$. The action will be homologically trivial (that is, induce the identity on integral homology), be pseudofree (that is, have only isolated fixed points) except in certain cases when $p=3$, and be locally linear except perhaps at one isolated fixed point. When $p=2$ and the intersection form of $M^{4}$ has even type, then the same conclusion holds. This is also due to S. Kwasik and P. Vogel [1985], by a somewhat different proof. If $p=2$ and the intersection form of $M$ has odd type, however, then an action of $\mathbf{Z}_{2}$ which is homologically trivial must have a fixed point set containing two-dimensional components (see $\S 7$ ). Further it is harder to control the Kirby-Siebenmann triangulation obstruction, which is then not determined by the intersection form. It is interesting to ask whether these actions can be locally linear or smooth (when $M^{4}$ is smooth). The existence of a locally linear $\mathbf{Z}_{2}$ action implies the vanishing of the Kirby-Siebenmann obstruction (see Kwasik and Vogel [1984]). We shall show, however, that (for $p>3$ in general) these actions can be constructed to be locally linear. In a recent preprint Kwasik has shown that this is the case for the fake $\mathbf{C} P^{2}$ when $p$ is odd by a rather different proof. These actions are homologically trivial and have only isolated fixed points (except sometimes when $p=3$ ). (The $G$-Signature Theorem shows that not every simply connected 4-manifold admits pseudofree, locally linear actions when $p=3$. We shall see, for example, that neither the $E_{8}$ manifold, the Kummer surface, nor a nontrivial connected sum of copies of $\mathbf{C} P^{2}$ admits such an action for $p=3$.)

In broad outline our construction goes as follows. Let a closed, oriented, simply connected 4-manifold $M$ be given. By studying equivariant framed links, we construct a compact smooth 4-manifold with boundary, having the intersection form of $M$, and admitting a smooth $\mathbf{Z}_{p}$ action which is pseudofree and homologically trivial. The action is free on the boundary homology sphere $\Sigma$. In a paper primarily focused on equivariant plumbing diagrams for high dimensional, highly connected manifolds with even intersection forms, Weintraub [1975] carried out most of this. We give an independent development because we need to be able to better control

Received by the editors October 16, 1985 and, in revised form, January 7, 1986.

1980 Mathematics Subject Classification (1985 Revision). Primary 57S17, 57S25, 57N15.

Research supported in part by a grant from the National Science Foundation. 
the Kirby-Siebenmann triangulation obstruction. We show (using an argument first noticed by D. Ruberman and, independently, Vogel and Kwasik [1985]) that any such free action can be extended to a topological action on a contractible 4 manifold $W$ with $\partial W=\Sigma$. In the case when the intersection form of $M$ has odd type one can change the original equivariant link to realize both a zero and a nonzero Kirby-Siebenmann invariant. One can analyze exactly when the action on $\Sigma$ can be extended to $W$ in a locally linear way. With care this locally linear extension can always be found (for suitable equivariant links) in the case of primes greater than three. The second construction for $p>3$ almost makes the first construction irrelevant. But it does show clearly how to construct many actions which cannot be locally linear.

The remainder of this paper is as follows. In $\S 1$ we describe the operation of equivariant surgery on an equivariant link for the cases we need. $\S 2$ is devoted to the algebra necessary to produce appropriate equivariant linking matrices. The topological extension theorem is proved in $\S 3$. In $\S 4$ we show how to vary the triangulation obstruction, and complete the argument for the first existence result. In $\S 5$ we investigate the problem of making these actions locally linear, which involves trying to replace the homology sphere $\Sigma$ mentioned in the sketch above with the standard 3-sphere with a standard free action. (The obstruction to doing this consists of an Atiyah-Singer signature invariant and a Reidemeister torsion.) In $\S 6$ we extend these arguments to construct locally linear actions on arbitrary simply connected 4-manifolds for any prime $p>3$, or, more generally, for any odd period not divisible by 3 . Finally, in $\S 7$ some observations are made concerning the problem of constructing involutions on manifolds with intersection form of odd type.

ACKnowledgment. Conversations with John Ewing, Slawomir Kwasik, Chuck Livingston, Danny Ruberman, Jim Davis, and Shmuel Weinberger were important at various stages of this work.

1. Equivariant surgery. Let $\mathbf{Z}_{p}$ act linearly on the 4-ball $D^{4}$, freely off of the origin 0 . (A convenient choice of this action, which we shall use particularly in $\S 4$, is the one naturally embedded in the Hopf circle action.) Let $K \subset S^{3}$ be an invariant, smooth, simple closed curve. Then the framings of the normal bundle of $K$ are indexed by the integers and each framing may be represented by an equivariant embedding $S^{1} \times D^{2} \rightarrow S^{3}$ for some standard action on $S^{1} \times D^{2}$. In particular, the "0-framing" is represented by an equivariant embedding $f_{0}: S^{1} \times D^{2} \rightarrow S^{3}$ such that $f_{0}\left(S^{1} \times\{0\}\right)=K, f_{0}\left(S^{1} \times\{1\}\right)$ has linking number 0 with $K$, and such that the induced action of $\mathbf{Z}_{p}$ on $S^{1} \times D^{2}$ is given by $g(z, w)=\left(\lambda^{a} Z, \lambda^{b} w\right)$, where $\lambda=e^{2 \pi i / p}$. It follows easily from covering space theory and the linking number condition that the integers $a$ and $b$, which are defined modulo $p$, cannot be $0 \bmod p$.

An arbitrary integer framing of $K$ may be represented by a map $f_{n}: S^{1} \times D^{2} \rightarrow$ $S^{3}$, where $f_{n}(z, w)=f_{0}\left(z, z^{n} w\right)$ for some integer $n$. Then $f_{n}$ is an equivariant map for the action of $\mathbf{Z}_{p}$ on $S^{1} \times D^{2}$ given by $g_{n}(z, w)=\left(\lambda^{a} z, \lambda^{b-n a} w\right)$.

Equivariant $n$-framed surgery on $K$ produces the 4-manifold $W^{4}=D^{4} \cup_{f_{n}} D^{2} \times$ $D^{2}$, where $D^{2} \times D^{2}$ has the action given by $g_{n}$. Note that $\mathbf{Z}_{p}$ acts by the identity on the homology of $W^{4}$ and has only isolated fixed points unless $b \equiv n a \bmod p$. In the latter case the fixed point set contains the 2-disk $\{0\} \times D^{2}$. The framings $n$ with $n a \equiv b \bmod p$ will be referred to as forbidden framings for the given 0 -framing 
action. The action given by $g_{n}$ will be called the $n$-framing action. The ordered pair $\{a, b-n a\}$ will be referred to as the type of the $n$-framing action. Note that the $n$-framing action depends only on $K$ and the congruence class of $n \bmod p$.

A completely similar construction applies to a framed link in $S^{3}$, each of whose components is invariant under the free $\mathbf{Z}_{p}$ action on $S^{3}$.

Let us look more closely at a specific example, a link in $S^{3}$ consisting of fibers in the Hopf fibering. Viewing $S^{3}$ as the unit sphere in $\mathbf{C}^{2}$, these fibers have the form $\left\{\left(\varsigma z_{0}, \zeta w_{0}\right): \varsigma \in S^{1}\right\}$. Every pair of fibers has linking number 1 . The actions of $\mathbf{Z}_{p}$ in the corresponding circle action yield the action $g(z, w)=(\lambda z, \lambda w)$ in the $S^{1} \times D^{2}$ coordinates given by the 0 -framing. In particular, the "forbidden" framings (for producing actions with only isolated fixed points) are those $n$ with $n \equiv 1 \bmod p$. Note that if one of the components of the link is altered by a $\mathbf{Z}_{p^{-}}$ equivariant isotopy (which is allowed to cross other components of the link), then the 0 -framing action remains the same, although the mutual linking numbers may be changed by multiples of $p$.

Observe that if invariant knots $K$ and $K^{+}$are equivariantly isotopic, then they have the same $n$-framing actions for all framings $n$. We will need the following lemma about framing actions and connected sums of knots.

LEMMA 1.1. Let $\mathbf{Z}_{p}$ act freely on a homology 3-sphere and let $K \subset \Sigma$ be an invariant knot. Let $K^{+}$be obtained by equivariantly inserting into $K p$ copies of another knot $K_{0}$ in $S^{3}$. Then for any framing $n$, the type of the $n$-framing action for $K^{+}$is the same as that for $K$.

ProOF. Let $Q=\Sigma / \mathbf{Z}_{p}$ and $\bar{K}$ (respectively $\bar{K}^{+}$) be the image of $K$ (respectively $\left.K^{+}\right)$in $Q$. Then $\left(Q, \bar{K}^{+}\right) \simeq(Q, \bar{K}) \#\left(S^{3}, K_{0}\right)$. Since all framings and framing actions may be induced from any given framing with its corresponding action, by standard self-maps of $S^{1} \times D^{2}$, it suffices to prove this result for any single given framing $n$. The simplest choice seems to be the forbidden framing $n$ that gives an action of type $\{a, 0\}$, which leaves the $n$-framing curve invariant. Clearly one can explicitly construct $K^{+}$so that its $n$-framing curve is invariant as well. The result follows.

It seems likely that Lemma 1.1 remains true if $K^{+}$is only assumed to be equivariantly homotopic into $K$.

We shall show (with certain exceptions for $p=2$ or 3 ) that for a symmetric bilinear form $\Phi: \mathbf{Z}^{m} \times \mathbf{Z}^{m} \rightarrow \mathbf{Z}$ of determinant \pm 1 there is a basis for $\mathbf{Z}^{m}$ with respect to which the matrix of $\Phi$ is the linking matrix of a framed link with all components invariant (and in fact unknotted) under the $\mathbf{Z}_{p}$ action on $S^{3}$ discussed above, with the 0 -framing action as above, and with no forbidden framings (in this case, those congruent to $1 \bmod p$ ).

2. The algebra of intersection forms. A key to the construction in this paper is that the complicated classification of integral bilinear forms simplifies considerably upon reduction modulo a prime $p$. The following fundamental result is known, but we include a sketch of a direct and elementary proof. In the case of odd primes it appears as a corollary of more general results in Hirzebruch, Neumann, and Koh [1971, 1.15]. 
LEMMA 2.1. A nonsingular, symmetric bilinear form over the field $\mathbf{Z}_{p}$ is determined up to equivalence by rank and determinant (the latter being understood modulo squares in $\mathbf{Z}_{p}$ ), and, in the case $p=2$, the type (odd or even).

ProOF. First suppose that $p \neq 2$. The form may certainly be diagonalized, so that with respect to a suitable basis it has a matrix which is an orthogonal sum $\left(a_{1}\right) \oplus \cdots \oplus\left(a_{n}\right)$, where the $a_{i}$ are units. If $a_{i}$ is a square, then a scaling change of basis changes $a_{i}$ to 1 ; if $a_{i}$ is not a square, then a scaling change of basis changes $a_{i}$ to some given nonsquare $\alpha$. Thus one may assume that the form has matrix (1) $\oplus \cdots \oplus(1) \oplus(\alpha) \oplus \cdots \oplus(\alpha)$. Consider the rank 2 form $\Psi$ with matrix $(\alpha) \oplus(\alpha)$. One can show that every element of $\mathbf{Z}_{p}$ is a sum of two squares; this easily shows there is an ordered pair $v=(a, b)$ such that $\Psi(v, v)=1$. It follows that there is a basis such that $\Psi$ is given by $(1) \oplus(\beta)$. Thus one eventually achieves a basis such that the given form has matrix $(1) \oplus \cdots \oplus(1) \oplus(\delta)$, where $\delta$ equals 1 or $\alpha$ and is equal to the determinant of the form modulo $p$ and squares in $\mathbf{Z}_{p}$. This completes the proof when $p$ is odd.

Now suppose that $p=2$. Let $\Phi$ be the given form, defined on $\left(\mathbf{Z}_{2}\right)^{n}$. If there is a vector $v$ such that $\Phi(v, v)=1$, then one may split off a one-dimensional orthogonal summand $\langle v\rangle$ by the Gram-Schmidt procedure. If $\Phi(v, v)=0$ for all $v$, then there are $v$ and $w$ such that $\Phi(v, w)=1$; and the Gram-Schmidt procedure shows that $\Phi$ is equivalent to a form $H \oplus \Psi$, where $H$ is the hyperbolic plane $\left[\begin{array}{ll}0 & 1 \\ 1 & 0\end{array}\right]$.

By induction, $\Phi$ has matrix $a(1) \oplus b H$ with respect to a suitable basis, where $a+2 b=n$. But the form (1) $\oplus H$ is easily seen to be equivalent to $3(1)$. It follows that an odd form is equivalent to $m(1)$, while an even form is equivalent to a sum of hyperbolic planes.

LEMMA 2.2. For any prime p, any nonsingular, symmetric bilinear form over the integers $\mathbf{Z}$ is equivalent to one whose reduction modulo $p$ is any given nonsingular, symmetric bilinear form over $\mathbf{Z}_{p}$ having the same rank and determinant modulo $p$, if $p$ is odd, and when $p=2$ having the type of the given form.

PROOF. This uses Lemma 2.1 plus the well-known surjectivity of the homomorphism $\mathrm{SL}_{m}(\mathbf{Z}) \rightarrow \mathrm{SL}_{m}\left(\mathbf{Z}_{p}\right)$ induced by reduction mod $p$. (Use elementary row operators to write a matrix in $\mathrm{SL}_{m}\left(\mathbf{Z}_{p}\right)$ as a product of elementary matrices of the form the identity plus one nonzero off-diagonal entry. Such matrices clearly lift.) First suppose that $p$ is odd. Let $A$ be an $m \times m$ symmetric integral matrix with determinant \pm 1 , and let $B$ b€ an $m \times m$ symmetric matrix with entries in $\mathbf{Z}_{p}$ such that $\operatorname{det} A \equiv \operatorname{det} B(\bmod p)$. According to Lemma 2.1 there is an $m \times m$ change of basis matrix $X$ over $\mathbf{Z}_{p}$ such that $X^{T} \bar{A} X \equiv B(\bmod p)$, where $\bar{A}$ denotes the reduction of $A \bmod p$. Then there is $Y \in \mathrm{SL}_{m}(\mathbf{Z})$ reducing to $X \bmod p$, and $Y^{T} A Y$ is the required integral matrix reducing to $B \bmod p$. The case $p=2$ is similar.

Compare Weintraub $[\mathbf{1 9 7 5}, \S 1]$. We now discuss some useful families of matrices. We note that at the expense of being somewhat less explicit one could refer to the results of $\S 6$ for the existence of matrices with the required properties, except when $p=2$ or $p=3$.

Proposition 2.3. Let $p>3$ be a prime, $m \geq 2$ be a positive integer, and $\varepsilon \in\{ \pm 1\}$. For integers $x_{1}, x_{2}, \ldots, x_{m}$ let $A=\left(a_{i j}\right)=A\left(m, 1 ; x_{1}, x_{2}, \ldots, x_{m}\right)$ be the $m \times m$ matrix with $a_{i i}=x_{i}$ and $a_{i j}=1$ for $i \neq j$. Then there exist $x_{i} \not \equiv 1$ $\bmod p, 1 \leq i \leq m$, such that the matrix $A$ has determinant $\varepsilon$ modulo $p$. 
Addendum for $p=3$. The proposition holds for $p=3$ provided that $m \geq 3$ or $m=2$ and $\varepsilon=-1$. It is easily seen to be false for $p=3, m=2$, and $\varepsilon=1$.

Addendum for $p=2$. If $p=2$ and $m$ is even, then $\operatorname{det} A(m, 1 ; 0, \ldots, 0) \equiv 1$ mod 2. (When $m$ is odd, the corresponding determinant is $0 \bmod 2$.)

We leave it to the reader to check the addendum for $p=2$. (Prove by induction on $m$ that, in the notation of Proposition $2.4, \operatorname{det} A(m, b ; x, \ldots, x)=$ $[x+(m-1) b](x-b)^{m-1}$.) To prove the results for odd primes we formulate a general proposition more amenable to a proof by induction.

Proposition 2.4. Let $p$ be an odd prime, $m \geq 2$ a positive integer (with $m \geq 3$ if $p=3$ ), $\varepsilon \in \mathbf{Z}_{p}^{*}$ (the nonzero residues $\bmod p$ ), and $b \in \mathbf{Z}_{p}$. Then there exist $x_{1}, x_{2}, \ldots, x_{m} \in \mathbf{Z}_{p}$ such that $x_{i} \neq b \bmod p, 1 \leq i \leq m$, and $\operatorname{det} A\left(m, b ; x_{1}, \ldots, x_{m}\right)$ $\equiv \varepsilon \bmod p$, where $A\left(m, b ; x_{1}, \ldots, x_{m}\right)$ is the $m \times m$ matrix $\left(a_{i j}\right)$ with $a_{i i}=x_{i}$ and $a_{i j}=b$ for $i \neq j$.

ProOF. We proceed by induction on $m$. Consider the case $m=2$. It is required to solve the equation $x_{1} x_{2}-b^{2}=\varepsilon$ in $\mathbf{Z}_{p}$, with neither $x_{1}$ nor $x_{2}$ equal to $b$. If $b=0$, then take $x_{1}=1, x_{2}=\varepsilon$. If $b \neq 0$ but $b^{2}+\varepsilon=0$, then take $x_{1}=x_{2}=0$. (These two cases work for $p=3$ as well.) If $b \neq 0$ and $b^{2}+\varepsilon \neq 0$, then there are $p-2$ ordered pairs $\left(x_{1}, x_{2}\right)$ with $x_{1} x_{2}=b^{2}+\varepsilon$ and $x_{1} \neq b$. At most one of these uses $x_{2}=b$. Therefore, there are at least $p-3$ solutions when $m=2$. (This last does not work for $p=3$.)

For $p=3$ we separately check the case $m=3$. (The situation when $m=2$ is left to the reader to analyze.) If $b=0$ this is easy. The cases $b=1$ and $b=-1$ are equivalent under the correspondences $1 \leftrightarrow-1$ and $\varepsilon \leftrightarrow-\varepsilon$. So suppose $b=1$. One may then check that $\operatorname{det} A(3,1 ; 0,0,0)=-1 \bmod 3$ and $\operatorname{det} A(3,1 ;-1,-1,-1)=1$ $\bmod 3$.

Now suppose inductively that $m>2(m>3$ if $p=3)$. If $b \neq-1$, set $x_{m}=-1$. Then using row operations to clear all entries in the last column except the bottom entry, we find that $\operatorname{det} A\left(m, b ; x_{1}, \ldots, x_{m}\right)=-\operatorname{det} A\left(m-1, b+b^{2} ; x_{1}+b^{2}, \ldots, x_{m-1}+\right.$ $\left.b^{2}\right)$. By induction we may choose $u_{1}, \ldots, u_{m-1}$ such that $u_{i} \neq b+b^{2} \bmod p$ and $\operatorname{det} A\left(m-1, b+b^{2} ; u_{1}, \ldots, u_{m}\right)=-\varepsilon \bmod p$. Set $x_{i}=u_{i}-b^{2}$. Then $x_{i} \neq b \bmod p$ and $\operatorname{det} A\left(m, b ; x_{1}, \ldots, x_{m}\right)=\varepsilon \bmod p$, as required.

Finally, if $b=-1 \bmod p$, set $x_{m}=1$. Then again using row operations to clear all entries in the last column except the bottom entry, we find that $\operatorname{det} A\left(m,-1 ; x_{1}, \ldots, x_{m}\right)=\operatorname{det} A\left(m-1,-2 ; x_{1}-1, \ldots, x_{m-1}-1\right)$. By induction there exist $u_{1}, \ldots, u_{m-1}$ with $u_{i} \neq-2 \bmod p$ such that

$$
\operatorname{det} A\left(m-1,-2 ; u_{1}, \ldots, u_{m-1}\right)=\varepsilon \text {. }
$$

Set $x_{i}=u_{i}+1$. Then $x_{i} \neq-1$ and $\operatorname{det} A\left(m,-1 ; x_{1}, \ldots, x_{m}\right)=\varepsilon$.

REMARK. Some related computations, given by Weintraub [1975] for plumbing matrices, provided important motivation for the preceding observations.

3. A topological extension theorem. The following proposition is the main result of this section.

Proposition 3.1. Let $\mathbf{Z}_{p}$ act freely on a homology 3 -sphere $\Sigma$. Then there is a contractible 4-manifold $N$ with $\partial N=\Sigma$ to which the action extends with exactly one isolated fixed point. 
We now proceed toward the proof of Proposition 3.1. Let $Q$ be the orbit space $\Sigma / \mathbf{Z}_{p}$.

LEMMA 3.2. There is an integer $q$ prime to $p$ for which there is a degree one map $f: Q \rightarrow L(p, q)$.

Proof. Note that $H^{1}\left(Q ; \mathbf{Z}_{p}\right) \approx \mathbf{Z}_{p}$. Let $x$ be a generator of this group. The Bockstein $\beta: H^{1}\left(Q ; \mathbf{Z}_{p}\right) \rightarrow H^{2}\left(Q ; \mathbf{Z}_{p}\right)$ is easily seen to be an isomorphism. Poincaré duality then implies that the cup product $x \cup \beta(x)$ is some multiple $q[Q]$ of the $\bmod p$ fundamental class $[Q]$, with $q$ nonzero $\bmod p$. Viewing the lens space $L=L(p, q)$ as the 3 -skeleton of a $K\left(\mathbf{Z}_{p}, 1\right)$ space, we may assume by cellular approximation that the classifying map $f: Q \rightarrow K\left(\mathbf{Z}_{p}, 1\right)$ for the regular covering $\Sigma \rightarrow Q$ maps $Q$ into $L(p, q)$. When $p$ is odd the $\bmod p$ cohomology $\operatorname{ring}$ of $K$ has the form $E(y) \otimes P(\beta(y))$, where $y$ is a one-dimensional class, $E(y)$ is the exterior algebra on $y$, and $P(y)$ is the $\bmod p$ polynomial algebra on the Bockstein of $y$. When $p=2$ the mod 2 cohomology algebra has the form $P(x)$, where $x$ is the generator in dimension one $\left(\right.$ and $\left.\beta(x)=x^{2}\right)$. We have $f^{*}(y)=x$, so that

$$
q[Q]=x \cup \beta(x)=f^{*}(y \cup \beta(y))=f^{*}(q[L])=q f^{*}[L],
$$

so that $[Q]=f^{*}[L](\bmod p)$. Here $[L]$ represents the fundamental class of $L$, and the relation $y \cup \beta(y)=q[L]$ is well known (compare Greenberg and Harper $[\mathbf{1 9 8 1}$, p. 295]). Thus we have a map $f: Q \rightarrow L$ of degree congruent to $1 \bmod p$. It is easy to change the degree of $f$ by any multiple of $p$, by a composition $Q \rightarrow Q \vee S^{3} \rightarrow$ $L \vee L \rightarrow L$. $\mathbf{Z}_{p}$.

LEMMA 3.3. There is a $\mathbf{Z}\left[\mathbf{Z}_{p}\right]$ h-cobordism $V$ with $\partial V=Q \cup-Q$ and $\pi_{1}(V)=$

Recall that to say that $V$ is a $\mathbf{Z}\left[\mathbf{Z}_{p}\right]$ h-cobordism means that $H *\left(V, Q ; \mathbf{Z}\left[\mathbf{Z}_{p}\right]\right)=0$, where the local coefficients are induced by the natural homomorphism $\pi_{1}(V) \rightarrow$ $H_{1}(V) \approx \mathbf{Z}_{p}$. This is equivalent to requiring that the $\mathbf{Z}_{p}$ cover be a $\mathbf{Z} h$-cobordism.

ProOF. Let $f: Q \rightarrow L$ be a degree one map, $L=L(p, q)$ as above. Then $f$ induces an isomorphism of integral homology and in fact of homology with coefficients in $\mathbf{Z}\left[\mathbf{Z}_{p}\right]$. Consider the map $g: Q \times I \rightarrow L \times I$ given by $g=f \times$ id, as a degree one normal map on which to attempt surgery relative to the boundary to obtain a homotopy equivalence. The surgery obstruction in $L_{\mathbf{4}}^{h}\left(\mathbf{Z}_{p}\right)$ is clearly zero, since it is the same as that to obtain a $\mathbf{Z}\left[\mathbf{Z}_{p}\right]$ homology equivalence (cf. Cappell and Shaneson $[\mathbf{1 9 7 4}])$, which we already have. According to Freedman [1983] Wall surgery theory for target manifolds with finite fundamental groups works in dimension 4 in the topological category. Then $V$ is the result of this surgery.

PROOF OF PROPOSITION 3.1. Let $V_{1}, V_{2}, \ldots, V_{n}, \ldots$ be a sequence of copies of the manifold $V$ guaranteed by Lemma 3.3. Let $X$ be the manifold $V_{1} \cup V_{2} \cup \ldots$ obtained by identifying the copy of $-Q$ in $\partial V_{i}$ with the copy of $Q$ in $\partial V_{i+1}$ for each positive integer $i$. By Van Kampen's theorem, $\pi_{1}(X) \approx \mathbf{Z}_{p}$. Let $Y$ be the universal covering of $X$. Then $\partial Y=\Sigma$ with the original group action. Moreover, $H_{2}(Y)=0, Y$ is simply connected, and the end of $Y$ is tame and simply connected. By results of Freedman the end of $Y$ is homeomorphic to $S^{3} \times \mathbf{R}$. Thus the one point compactification of $Y$ is the required manifold $N$, to which the group of covering transformations extends fixing the added point. 
REMARKS. In the case $p=2$, a version of this argument was first shown to me by $D$. Ruberman. It was also known in this case to Kwasik and Vogel. In $\S 5$ we shall address the question of when the extension of Proposition 3.1 can be done locally linearly.

4. The topological existence theorem. This section is devoted to the proof of the first existence result for actions on 4-manifolds.

THEOREM 4.1. Let $M^{4}$ be a closed, simply connected topological 4-manifold and $p$ be a prime. If $p$ is odd or if $p=2$ and the intersection form of $M$ has even type, then $M^{4}$ admits an action of $\mathbf{Z}_{p}$ that is homologically trivial, pseudofree, and locally linear except perhaps at one isolated fixed point. (In one exceptional case when $p=3$ and $M$ has the homotopy type of $\mathbf{C P}^{2} \# \mathbf{C} P^{2}$, the fixed point set contains a 2-sphere.)

According to Freedman, closed, simply connected 4-manifolds are classified up to homeomorphism by their intersection forms, and, in the case of odd forms, the Kirby-Siebenmann triangulation obstruction in $H^{4}\left(M ; \mathbf{Z}_{2}\right) \approx \mathbf{Z}_{2}$. Any unimodular form and (in the case of odd forms) a zero or nonzero triangulation obstruction may be realized.

First consider the case that $p>3$. Let $\Phi: \mathbf{Z}^{m} \times \mathbf{Z}^{m} \rightarrow \mathbf{Z}$ be the intersection form of $M^{4}$. If $m>1$, then the results of $\S 2$ imply that, with respect to a suitable basis, $\Phi$ has a matrix $A=\left(a_{i j}\right)$ which reduces $\bmod p$ to the matrix $A\left(m, 1 ; x_{1}, \ldots, x_{m}\right)$ of Proposition 2.3, with no $x_{i} \equiv 1 \bmod p$. If $m=1$, we may, by a reversal of orientation if necessary, assume that $\operatorname{det} \Phi=-1$; then let $A=(-1)$. Let $K$ be the link in $S^{3}$ which consists of $m$ fibers of the right-hand Hopf fibering, with framings $a_{i i}, 1 \leq i \leq m$. This link is invariant under the standard $\mathbf{Z}_{p}$ action described in $\S 2$.

For each component of $K$ the forbidden framings, for which equivariant surgery leads to a two-dimensional fixed point set, are those congruent to $1 \bmod p$, so none of the framings $a_{i i}$ are forbidden (since $p \neq 2,3$ ). By equivariant isotopies of the various components of $K$, which push a small arc on one component and its $p-1$ translates across another component, one may alter the off-diagonal entries of the linking matrix by arbitrary multiples of $p$, maintaining symmetry.

Thus we arrive at a framed link $K$ with linking matrix $A$ which is invariant under a free action of $\mathbf{Z}_{p}$ on $S^{3}$, such that none of the framings are forbidden.

Equivaraint surgery on $K$ produces a compact, simply connected, smooth 4manifold $W^{4}$ which has intersection form given by the matrix $A$ and which has a smooth $\mathbf{Z}_{p}$ action inducing the identity on homology and having only isolated fixed points in the interior of $W^{4}$. Since $A$ is a unimodular matrix, duality implies that $\partial W^{4}$ is a homology sphere. Then by Proposition 3.1 the action on $\partial W^{4}$ extends to one on a contractible manifold $N^{4}$ with $\partial N^{4}=\partial W^{4}$. Then $M_{1}=W^{4} \cup_{\partial} N^{4}$ is a closed, simply connected 4-manifold with intersection form equivalent to $\Phi$, admitting a $\mathbf{Z}_{p}$ action which is the identity on homology, which has only isolated fixed points, and which is locally linear except perhaps at one point. According to Freedman, $M_{1}$ is homeomorphic to $M^{4}$, except perhaps when $\Phi$ has odd type, in which case $M^{4}$ and $M_{1}$ may have different Kirby-Siebenmann classes. It suffices in this case to show how to alter the link $K$ to reverse the Kirby-Siebenmann class. 
The framed link $K$ contains a characteristic sublink $K_{0}$ (such that if $K_{1}$ is a component of $K$, then the framing of $K_{1}$ is the linking number $\left.\mathbf{l k}\left(K_{1}, K_{0}\right)(\bmod 2)\right)$. The triangulation obstruction for the resulting 4-manifold will change if one inserts into a component of $K_{0}$ a small trefoil (with framing 0 ), hence also if one inserts $p$ such small trefoils (since $p$ is odd). (In Freedman [1982, p. 371], it is assumed that $K_{0}$ is connected, but this is clearly unnecessary.) This can be done equivariantly to obtain a framed link $K^{\prime}$ with the same linking matrix as $K$. It follows from Lemma 1.1 that the framing actions on tubes about the components of $K^{\prime}$ are not forbidden, since those for $K$ are not.

Now assume $p=2$ and that the intersection form of $M^{4}$ has even type. It follows that the rank of $H_{2}(M)$ is even. The argument then proceeds just as in the case $p$ is odd, using the matrix $A(m, 1 ; 0, \ldots, 0)$, except that the triangulation obstruction is determined completely by the intersection form.

It remains to consider the case $p=3$. Unless $m=2$, and $\operatorname{det} \Phi=1$ the first addendum to Proposition 2.3 shows that the proof given above works. Suppose, however, that $p=3, m=2$, and $\operatorname{det} \Phi=1$. In this case there is no $2 \times 2$ matrix for $\Phi$ that does not have +1 on the diagonal! Up to a change of orientation there are exactly two manifolds to consider: $\mathbf{C} P^{2} \# \mathbf{C} P^{2}$ and $\mathrm{Ch} \# \mathbf{C} P^{2}$, where $\mathrm{Ch}$ denotes the "Chern manifold," the unique topological 4-manifold that is homotopy equivalent, but not homeomorphic, to $\mathbf{C} P^{2}$. Now $\mathbf{C} P^{2}$ admits standard linear $\mathbf{Z}_{3}$ actions with three isolated fixed points, and (by the previously discussed cases) Ch admits a $\mathbf{Z}_{3}$ action with three isolated fixed points at least two of which are locally linear. One may not construct the (oriented) equivariant connected sum of two of these actions because all the local representations at isolated fixed points are exactly the same, given by the pair of rotation angles $(2 \pi / 3,4 \pi / 3)$. (Compare Lemma 5.4.) There is another standard action on $\mathbf{C} P^{2}$ fixing $\mathbf{C} P^{1} \cup \mathbf{C} P^{0}$, with the local representation at the isolated fixed point given by $(2 \pi / p, 2 \pi / p)$. One may now construct the equivariant connected sum $\mathbf{C} P^{2} \#$ Ch by taking the action with a two-dimensional fixed point set on the first summand. Similarly one constructs the required action on $\mathbf{C} P^{2} \# \mathbf{C} P^{2}$.

REMARK. It follows easily from the $G$-signature theorem that neither $\mathbf{C} P^{2} \#$ $\mathbf{C} P^{2}$ nor $\mathbf{C} P^{2}$ \# Ch admits a locally smooth $\mathbf{Z}_{3}$ action which is the identity on homology and has only isolated fixed points. Is there a purely topological action?

REMARK. If $M^{4}$ has an odd intersection form and $p=2$, then one may follow the first part of the preceding argument, using the matrix $A(m, 1 ; 1,0, \ldots, 0)$, to produce a smooth $\mathbf{Z}_{2}$ action on a compact, simply connected manifold $W^{4}$ whose intersection form is equivalent to that of $M^{4}$, such that $\mathbf{Z}_{2}$ acts by the identity on homology, with fixed point set consisting of isolated points, except for one component which is a 2-disk dictated by the forbidden framing encountered. One is left with two questions: Can an involution on a homology 3 -sphere which fixes a simple closed curve be extended to an involution on a contractible 4-manifold? Can one effectively control the triangulation obstruction?

5. A locally linear extension theorem. In this section we investigate the problem of carrying out the construction of $\S 4$ in such a way as to produce a locally linear action on a given 4-manifold, acting by the identity on homology and having only isolated fixed points. The fundamental problem turns out to be one of deciding in an effective way when a 3-dimensional $\mathbf{Z}\left[\mathbf{Z}_{p}\right]$ homology lens space is 
$\mathbf{Z}\left[\mathbf{Z}_{p}\right] h$-cobordant to a classical lens space. This is accomplished by transferring Wall's classification of fake lens spaces in higher dimensions to the 3-dimensional situation.

THEOREM (WALL [1970, §14]). Two homotopy lens spaces (of dimension five or higher) with odd order fundamental group $\mathbf{Z}_{p}$ are homeomorphic if and only if they have the same $\alpha$-invariants and equivalent Reidemeister torsions.

We will explain the terms " $\alpha$-invariant" and "Reidemeister torsion" below; for the moment it suffices to know that they are certain algebraic integers in $\mathbf{Q}\left[e^{2 \pi i / p}\right]$. Moreover, these two invariants are perfectly well defined for a homology lens space occurring in dimension three as the quotient of a homology 3 -sphere by a free $\mathbf{Z}_{p}$ action. Wall describes which invariants are realizable, as well as the precise relationship between them. If two homotopy lens spaces are $h$-cobordant, then they have the same $\alpha$-invariants, but their Reidemeister torisons differ by a factor of the square of the Whitehead torsion of the $h$-cobordism. Thus we may apply Wall's classification theorem, Milnor's duality theorem for Reidemeister torsion, and the realizability of Whitehead torsion by $h$-cobordisms to prove the following result.

COROLLARY. Two homotopy lens spaces (of dimension five or higher) with odd order fundamental group $\mathbf{Z}_{p}$ are $h$-cobordant if and only if they have the same $\alpha$ invariants, and their Reidemeister torsions differ, up to equivalence, by the square of a unit of $\mathbf{Z}\left[e^{2 \pi i / p}\right]$ that has augmentation congruent to 1 modulo $p$.

We now transfer Wall' theory to the 3-dimensional setting by applying Freedman' results. Let $\Sigma$ be a homology 3 -sphere with free $\mathbf{Z}_{p}$ action, and let $Q=\Sigma / \mathbf{Z}_{p}$. Let $L=L(p, q)$ be the lens space such that there is a $\mathbf{Z}\left[\mathbf{Z}_{p}\right]$ homology equivalence $f: Q \rightarrow L$ inducing a preferred isomorphism of fundamental groups, covered by an equivariant $\mathbf{Z}$ homology equivalence $\Sigma \rightarrow S^{3}$. Because 3-manifolds are parallelizable, we may view the map $f$ as a normal map, covered by a bundle map $b: \nu(Q) \rightarrow \nu(L)$.

The set of smooth (or equivalently piecewise linear) normal cobordism classes of normal maps to $L$ is in one-to-one correspondence with the set of homotopy classes $[L ; G / \mathrm{PL}]$. But since $L$ is 3 -dimensional, $[L ; G / \mathrm{PL}]=0$ for $p$ odd. (Compare Wall $[\mathbf{1 9 7 0}$, p. 210]). Therefore there is a normal cobordism $F:(V ; Q, L) \rightarrow$ $(L \times[0,1] ; L \times\{0\}, L \times\{1\}]$ (covered by a suitable bundle map $B$ ) from $f: Q \rightarrow L$ to the identity $1_{L}: L \rightarrow L$. By surgery on circles in $V$ one may assume that $\pi_{1}(V)=\mathbf{Z}_{p}$.

The Wall obstruction $\theta(F) \in L_{4}^{h}\left(\mathbf{Z}_{p}\right)$ is detected by two invariants: the $g$ $\operatorname{signature} \operatorname{sign}(g, \tilde{V})$ of the deck transformation on the $\mathbf{Z}_{p}$ cover of $V$ and a ratio $\Delta(Q) / \Delta(L)$ of Reidemeister torsions. (If $f: Q \rightarrow L$ were a simple $\mathbf{Z}\left[\mathbf{Z}_{p}\right]$ homology equivalence, then the surgery obstruction would live in $L_{4}^{s}\left(\mathbf{Z}_{p}\right)$, and no discussion of Reidemeister torsions would be required.)

The $g$-signature $\operatorname{sign}(g, \tilde{V})$ can be expressed as the difference of two $\alpha$-invariants (or "multisignatures") $\alpha(L)-\alpha(Q)$, defined by Atiyah and Bott for any evendimensional manifold $M$ with preferred homomorphism $\pi_{1} M \rightarrow \mathbf{Z}_{p}$ using the $G$ Signature Theorem, as follows. The covering $\Sigma \rightarrow Q$ is classified by a map $Q \rightarrow$ $K\left(\mathbf{Z}_{p}, 1\right)$. Since the oriented bordism group $\Omega_{3}\left(K\left(\mathbf{Z}_{p}, 1\right)\right) \otimes \mathbf{Q}=0$, some integral 
multiple $r Q$ of $Q$ bounds over $K\left(\mathbf{Z}_{p}, 1\right)$. In other words there is a manifold $X$ with $\partial X=r \Sigma$ having a free $\mathbf{Z}_{p}$ action extending that on $r \Sigma$. Let $g: X \rightarrow X$ denote the generator of the action. We set $\alpha(Q)=\operatorname{sign}(g, X) / r$. This is easily seen to be independent of the value of $r$ chosen, and is well defined since the $g$-signature is zero for free actions on closed manifolds. The fact that $\operatorname{sign}(g, \tilde{V})=\alpha(L)-\alpha(Q)$ follows for the same reason. Clearly this invariant exists for any 3-manifold with homomorphism of its fundamental group onto $\mathbf{Z}_{p}$.

To compute $\alpha(Q)$ one need not restrict attention to free actions; one may apply the $G$-Signature Theorem to correct for the presence of fixed points. (It follows from results of Wall $[\mathbf{1 9 7 0}, \S 14 \mathrm{~B}]$, and Freedman $[\mathbf{1 9 8 3}]$ that the $G$-Signature Formula remains valid for locally linear actions on topological 4-manifolds.) Thus, if $X$ is any oriented manifold with locally linear $\mathbf{Z}_{p}$ action, and $\partial X=\Sigma$ as oriented manifolds with group actions, then

$$
\alpha(Q)=\operatorname{sign}(g, X)-\sum \mathbf{e}\left(F^{2}\right) \operatorname{cosec}^{2}\left(\psi\left(F^{2}\right) / 2\right)+\sum \cot \left(\theta_{1}(P) / 2\right) \cot \left(\theta_{2}(P) / 2\right) .
$$

Here the two sums run over the two-dimensional components $F^{2}$ of the fixed point set and the isolated fixed points $P$ in $X$, respectively. The Euler number of the normal bundle of $F^{2}$ is denoted $\mathbf{e}\left(F^{2}\right) ; \psi\left(F^{2}\right)$ denotes the rotation angle for $g$ in the normal bundle of $F^{2}$; and $\theta_{1}(P), \theta_{2}(P)$ denote the rotation angles for $g$ near $P$ (well defined up to order or changing the sign of both). The $G$-Signature Theorem says that $\alpha(Q)$ is well defined. It follows, for example, that if $L(p ; a, b)$ denotes the standard lens space arising from the linear action of $\mathbf{Z}_{p}$ on $\mathbf{C}^{2}$ of type $\{a, b\}$, then $\alpha(L(p ; a, b))=\cot (a \pi / p) \cot (b \pi / p)$. One may use $\alpha$-invariants to classify standard lens spaces up to homeomorphism.

We now discuss the Reidemeister torsion $\Delta(Q)$ of the $\mathbf{Z}\left[\mathbf{Z}_{p}\right]$ homology lens space $Q$, again understood to have a preferred homomorphism of $\pi_{1}(Q)$ onto $\mathbf{Z}_{p}$. The basic reference is Milnor [1966]. Let $Q$ be given a cell structure (arising, for example, from a triangulation) and let $\Sigma$ be given the induced cell structure. The cellular chain complex $C_{*}(\Sigma)$ is then a complex of free $\mathbf{Z}\left[\mathbf{Z}_{p}\right]$ modules with a class of preferred bases determined by the cell structure. There are natural homomorphisms $\mathbf{Z}\left[\mathbf{Z}_{p}\right] \rightarrow \mathbf{Z}[\lambda] \rightarrow \mathbf{Q}[\lambda]$, where $\lambda=e^{2 \pi i / p}$, and $C_{*}(\Sigma) \otimes \mathbf{Q}[\lambda]$ is an acyclic complex of free based $\mathbf{Q}[\lambda]$ modules. This complex then has a torsion $\tau \in K_{1}(\mathbf{Q}[\lambda])$. But $K_{1}(\mathbf{Q}[\lambda])$ may be identified with the units $\mathbf{Q}[\lambda]^{\times}$of the ring $\mathbf{Q}[\lambda]$. Then $\Delta(Q)$ is the element of $\mathbf{Q}[\lambda]^{\times}$corresponding to the torsion $\tau$. The symbol $\Delta$ is used to signify multiplicative notation, as opposed to $\tau$ which connotes additive notation. We also adopt the convention that $\Delta_{1} \sim \Delta_{2}$ if $\Delta_{2}= \pm \lambda^{i} \Delta_{1}$ for some $i$. As a specific computation one may verify that $\Delta(L(p ; a, b)) \sim\left(\lambda^{a}-1\right)\left(\lambda^{b}-1\right)$. One may use Reidemeister torsion to classify standard lens spaces up to homeomorphism.

Corresponding to Wall's classification of fake lens spaces in higher dimensions we have the following classification of homology lens spaces in dimension 3 up to $\mathbf{Z}\left[\mathbf{Z}_{p}\right] s$-cobordism.

PROPOSITION 5.1. Let $p$ be an odd prime and let a three-dimensional $\mathbf{Z}\left[\mathbf{Z}_{p}\right]$ homology lens space $Q$ be given. Then $Q$ is $\mathbf{Z}\left[\mathbf{Z}_{p}\right]$ s-cobordant to a classical lens space $L=L(p, q)$ if and only if $\alpha(Q)=\alpha(L)$ and $\Delta(Q) \sim \Delta(L)$.

PROOF SKETCH. Note that $L$ is uniquely determined by $Q$ with its preferred homomorphism $\pi_{1}(Q) \rightarrow \mathbf{Z}_{p}$, as in Lemma 3.2 . If $Q$ and $L$ are just $\mathbf{Z}\left[\mathbf{Z}_{p}\right] h$ cobordant, then one easily sees from the comments above and the $G$-Signature 
Theorem that $\alpha(Q)=\alpha(L)$. In this case it further follows from considerations of duality, as in Milnor [1966, Theorem 12.8], that $\Delta(Q) \sim u^{2} \Delta(L)$, where $u$ is the image in $\mathbf{Q}[\lambda]$ of the Whitehead torsion of the $h$-cobordism. (Since $u$ corresponds to a unit of $\mathbf{Z}\left[\mathbf{Z}_{p}\right], u$ is a unit of $\mathbf{Z}[\lambda]$ of augmentation in $\mathbf{Z}_{p}$ congruent to \pm 1 .) This proves the necessity of the two conditions.

Conversely, suppose that $\alpha(Q)=\alpha(L)$ and $\Delta(Q) \sim \Delta(L)$. Then, applying Lemma 3.2 and the discussion above, there is a degree one map $f: Q \rightarrow L$ that is a $\mathbf{Z}\left[\mathbf{Z}_{p}\right]$ homology equivalence inducing the identity on $H_{1}$ (identified with $\mathbf{Z}_{p}$ in each case) and is normally cobordant to the identity $L \rightarrow L$. The map $f$ is a simple $\mathbf{Z}\left[\mathbf{Z}_{p}\right]$ homology equivalence, by Milnor [1966, Lemma 12.5]. Therefore the normal cobordism has a well-defined surgery obstruction in $L_{4}^{s}\left(\mathbf{Z}_{p}\right)$ that may be assumed to vanish by the hypothesis on $\alpha$-invariants. Applying Freedman [1983], one may perform surgery to obtain a normal cobordism $(V ; Q, L)$ with $\pi_{1}(V) \approx \mathbf{Z}_{p}$ which maps by a simple $\mathbf{Z}\left[\mathbf{Z}_{p}\right.$ homology equivalence to $(L \times[0,1], L \times\{0\}, L \times\{1\})$. Then $V$ is a $\mathbf{Z}\left[\mathbf{Z}_{p}\right]$ homology $s$-cobordism, as required.

COROLlaRY 5.2. If $p$ is an odd prime, then a three-dimensional $\mathbf{Z}\left[\mathbf{Z}_{p}\right]$ homology lens space $Q$ is $\mathbf{Z}\left[\mathbf{Z}_{p}\right]$ homology h-cobordant to a classical lens space $L$ if and only if $\alpha(Q)=\alpha(L)$ and $\Delta(Q) \sim u^{2} \Delta(L)$, where $u$ is the image in $\mathbf{Q}[\lambda]$ of a unit of $\mathbf{Z}\left[\mathbf{Z}_{p}\right]$.

PROOF. The necessity of the conditions is included in the preceding proof. Suppose now that the two conditions hold. The usual proof of the realization of Whitehead torsions by $h$-cobordisms in high dimensions may be modified to show that there is a $\mathbf{Z}\left[\mathbf{Z}_{p}\right]$ homology $h$-cobordism $\left(W ; Q, Q^{\prime}\right)$ with torsion $\tau \in \mathrm{Wh}\left(\mathbf{Z}_{p}\right)$ corresponding to $u$. Then $Q^{\prime}$ is a $\mathbf{Z}\left[\mathbf{Z}_{p}\right]$ homology lens space, and by Milnor $[\mathbf{1 9 6 6}$, Theorem 12.8], we have $\Delta\left(Q^{\prime}\right) \sim u^{2} \Delta(Q)$. Moreover, $\alpha\left(Q^{\prime}\right)=\alpha(Q)$, so that $Q^{\prime}$ is $\mathbf{Z}\left[\mathbf{Z}_{p}\right]$ homology $s$-cobordant to $L$. Concatenation of the two homology $h$ cobordisms proves the corollary.

PROPOSITION 5.3. If $p$ is an odd prime, then a free action of $\mathbf{Z}_{p}$ on a homology 3-sphere $\Sigma$ extends to a locally linear action on a contractible manifold $N$ with $\partial N=\Sigma$ if and only if the $\alpha$-invariant of $\Sigma / \mathbf{Z}_{p}$ equals the $\alpha$-invariant of some lens space $L$, and $\Delta\left(\Sigma / \mathbf{Z}_{p}\right) \sim u^{2} \Delta(L)$, where $u$ is the image in $\mathbf{Q}[\lambda]$ of a unit of $\mathbf{Z}\left[\mathbf{Z}_{p}\right]$.

PROOF. First suppose the action on $\Sigma$ does extend locally linearly to a contractible manifold $N$. Let $D$ be a small, flat invariant ball on which the action is equivalent to a linear action. Let $W=\overline{N-D}$. One easily sees that $W / \mathbf{Z}_{p}$ is a $\mathbf{Z}\left[\mathbf{Z}_{p}\right]$ homology $h$-cobordism from $\Sigma / \mathbf{Z}_{p}$ to a classical lens space. The corollary implies the conclusion.

Now suppose that the action on $\Sigma$ satisfies the hypotheses on $\alpha$-invariants and Reidemeister torsions. The corollary implies that there is a $\mathbf{Z}\left[\mathbf{Z}_{p}\right]$ homology $h$ cobordism $V$ from $\Sigma / \mathbf{Z}_{p}$ to a classical lens space $L$. Let $W$ be the corresponding $p$-fold covering of $V$ with deck transformation group $\mathbf{Z}_{p}$. Then $W$ is a $\mathbf{Z}$ homology cobordism from $\Sigma$ to $S^{3}$. We need to arrange for $W$ to be simply connected, or equivalently $\pi_{1}(V) \approx \mathbf{Z}_{p}$. Then we can cone off the linear action on $S^{3}$ to get the required action on a contractible (as opposed to just acyclic) manifold. Now $V$ was obtained as the result of surgery on a normal map to $L \times I$ with vanishing surgery obstruction, concatenated with a suitable $\mathbf{Z}\left[\mathbf{Z}_{p}\right]$ homology $h$-cobordism with prescribed torsion. In particular there is a normal map $V \rightarrow L \times I$ which 
clearly has vanishing absolute surgery obstruction in $L_{4}^{h}\left(\mathbf{Z}_{p}\right)$. Perform preliminary surgeries to obtain a normal map $V^{\prime} \rightarrow L \times I$, where $\pi_{1}\left(V^{\prime}\right) \approx \mathbf{Z}_{p}$. Then apply Freedman $[\mathbf{1 9 8 3}]$ to surger 2 -spheres to obtain a homotopy equivalence $V^{\prime \prime} \rightarrow L \times I$, as required.

6. The locally linear existence theorem. In this section we shall show that if $p$ is a prime greater than three, then $\mathbf{Z}_{p}$ acts locally linearly with only isolated fixed points on any closed, simply connected 4-manifold $M$, inducing the identity on $H_{2}(M ; \mathbf{Z})$. We begin with a discussion of some model actions on connected sums of copies of $\mathbf{C} P^{2}$ and $\overline{\mathbf{C} P^{2}}$.

LEMMA 6.1. Let $p$ be an odd prime and $a$ and $b$ be integers which are nonzero and distinct $\bmod p$. Then there is a smooth map $T: \mathbf{C} P^{2} \rightarrow \mathbf{C} P^{2}$ of period $p$ with three isolated fixed points at which the induced actions on the tangent spaces have types $\{a, b\},\{a-b,-b\}$, and $\{b-a,-a\}$.

Proof. Define $T$ in homogeneous coordinates by $T\left(z_{0}, z_{1}, z_{2}\right)=\left(\lambda^{a} z_{0}, \lambda^{b} z_{1}, z_{2}\right)$.

Note that the same action, when viewed on $\overline{\mathbf{C} P^{2}}$, has fixed points of types $\{a,-b\},\{a-b, b\}$, and $\{b-a, a\}$. The standard Morse function $f: \mathbf{C} P^{2} \rightarrow \mathbf{R}$ defined by $f\left(z_{0}, z_{1}, z_{2}\right)=\left|z_{1}\right|^{2}+2\left|z_{2}\right|^{2}$ shows that $\mathbf{C} P^{2}$ with this action may be described as being obtained from the 4-ball $D^{4}$ with a standard linear action of type $\{a, b\}$ by adding a 2-handle along an invariant simple closed curve (as described in $\S 1$, and adding a linear 4-handle along the resulting 3 -sphere boundary with linear action.

LEMMA 6.2. Let $p$ be a prime greater than three and let $M$ be the connected sum of $m$ copies of $\mathbf{C} P^{2}$ and $n$ copies of $\overline{\mathbf{C} P^{2}}$. Then $M$ admits a smooth, pseudofree, homologically trivial action of $\mathbf{Z}_{p}$.

PROOF. The argument is by induction on $m+n$. Lemma 6.1 starts the induction. Inductively we may assume (by reversal of orientation if necessary) that $M=$ $N \# \mathbf{C} P^{2}$ and that $N$ admits a smooth, pseudofree, homologically trivial action of $\mathbf{Z}_{p}$. Additionally assume that the action on $N$ does not have all its fixed point types of the form $\{x,-x\}$. This holds for the standard actions on $\mathbf{C} P^{2}$ and $\overline{\mathbf{C} P^{2}}$ above, provided that $p>3$. We show that $M$ admits such an action.

Let $P$ be a fixed point of the action on $N$ of type $\{x, y\}$, where $y \neq-x(\bmod p)$. Consider a standard action on $\mathbf{C} P^{2}$ with fixed points of types $\{x,-y\},\{x+y, y\}$, and $\{y+x, x\}$, as given by Lemma 6.1. Let $Q$ be the fixed point of type $\{x,-y\}$ in $\mathbf{C} P^{2}$. One may then construct the connected sum $N \# \mathbf{C} P^{2}$ in an equivariant way, yielding the required action.

Consideration of a standard Morse function on $M$ above shows that these model actions may also be described as being obtained by equivariant surgery on an appropriate framed link, each component of which is invariant under a certain linear action on $S^{3}$, capping off the resulting linear action on the final boundary 3-sphere wth a linear 4-handle.

Addendum for $p=3$. Suppose that $M$ is a closed, simply connected 4-manifold with a locally linear $\mathbf{Z}_{3}$ action that is pseudofree and homologically trivial. Then the signature defect formula (see Hirzebruch and Zagier [1973], for example), says that $2 \sigma=2 x / 3$, where $\sigma$ is the signature of $M$ (= the signature of the orbit space), and $x$ 
is the number of fixed points of type $\{1,2\}$ minus the number of type $\{1,1\}$. Now $|x|$ is at most the total number of fixed points, which is $\chi(M)=\beta_{2}(M)+2$. Therefore we must have $3|\sigma| \leq \beta_{2}(M)+2$, or $|\sigma| \leq\left(\beta_{2}(M)+2\right) / 3$. This shows that many manifolds do not support such $\mathbf{Z}_{3}$ actions: the $E_{8}$ manifold, the Kummer surface, a connected sum of $n>1$ copies of $+\mathbf{C} P^{2}$ or $+\mathrm{Ch}$, etc. For a connected sum of copies of $\mathbf{C} P^{2}$ and $\overline{\mathbf{C} P^{2}}$, it is not hard to see that the inequality $|\sigma| \leq\left(\beta_{2}(M)+2\right) / 3$ is in fact sufficient to construct (by equivariant connected sums) a locally linear, pseudofree, homologically trivial action of $\mathbf{Z}_{p}$. The proof of Theorem 6.4 shows that this last statement holds true for any closed, simply connected 4-manifold.

We shall need the following technical lemma about the behavior of Reidemeister torsion under equivariant surgery.

LEMMA 6.3. Let $\mathbf{Z}_{p}$ act smoothly on the homology 3-sphere $\Sigma$. Let $K^{+}=$ $K_{1}^{+} \cup K_{2}^{+} \cup \cdots \cup K_{m}^{+}$and $K^{-}=K_{1}^{-} \cup K_{2}^{-} \cup \cdots \cup K_{m}^{-}$be two framed links of $m$ components, with unimodular linking matrices, each component invariant under the $\mathbf{Z}_{p}$ action. Suppose that for $i=1, \ldots, m$ the framing action for $K_{i}^{+}$is the same as that for $K_{i}^{-}$and is not forbidden. Let $\Sigma^{+}$and $\Sigma^{-}$denote the results of equivariant surgery on the two framed links. Then $\Delta\left(\Sigma^{+} / \mathbf{Z}_{p}\right) \sim \Delta\left(\Sigma^{-} / \mathbf{Z}_{p}\right)$.

Proof. Let $Q, Q^{+}$, and $Q^{-}$denote the quotients of $\Sigma, \Sigma^{+}$, and $\Sigma^{-}$by $\mathbf{Z}_{p}$. Let $\bar{K}^{ \pm}$denote the image in $Q$ of $K^{ \pm}$; and denote by $X\left(\bar{K}^{ \pm}\right)$the exterior of $\bar{K}^{ \pm}$in $Q$, that is, $Q$ minus the interior of a small regular neighborhood of $\bar{K}^{ \pm}$. Let the action of $\mathbf{Z}_{p}$ on $K_{i}^{ \pm}$be given by rotation by $2 \pi r_{i} / p$; and the framing action on $K_{i}^{ \pm}$ be of type $\left\{r_{i}, s_{i}\right\}$, where $s_{i}$ is nonzero $\bmod p$ by the hypothesis of no forbidden framings.

First note that $\Delta(Q) \sim\left(\lambda^{r_{1}}-1\right) \cdots\left(\lambda^{r_{m}}-1\right) \Delta\left(X\left(\bar{K}^{ \pm}\right)\right)$. To see this, observe that the action induced on the homology of $\Sigma-K^{ \pm}$is clearly trivial, implying that $\Delta\left(X\left(\bar{K}^{ \pm}\right)\right)$is defined. The asserted formula then follows easily from seeing how the 2-cells and 3-cells are attached to a cell complex for $X\left(\bar{K}^{ \pm}\right)$to build up $Q$.

Next note that $\Sigma$ is obtained from $\Sigma^{ \pm}$by equivariant surgery on a complementary invariant link $K^{* \pm}$ with $X\left({\overline{K^{*}}}^{ \pm}\right)=X\left(\bar{K}^{ \pm}\right)$, such that on the typical component $\tilde{K}_{i}^{ \pm}$the action is given by rotation by $2 \pi s_{i} / p$. Thus we also have

$\Delta\left(Q^{ \pm}\right) \sim\left(\lambda^{s_{1}}-1\right) \cdots\left(\lambda^{s_{m}}-1\right) \Delta\left(X\left(\overline{K^{*}} \pm\right)\right) \sim\left(\lambda^{s_{1}}-1\right) \cdots\left(\lambda^{s_{m}}-1\right) \Delta\left(X\left(\bar{K}^{ \pm}\right)\right)$.

From this it follows that $\Delta\left(Q^{+}\right) \sim \Delta(Q) \prod_{i}\left(\lambda^{s_{i}}-1\right) /\left(\lambda^{r_{i}}-1\right)=\Delta\left(Q^{-}\right)$.

THEOREM 6.4. Let $p>3$ be a prime and $M$ be a closed, simply connected 4-manifold. Then there is a locally linear, pseudofree, homologically trivial action of $\mathbf{Z}_{p}$ on $M$.

Proof. Let $m=\beta_{2}(M)$ be the rank of $H_{2}(M)$ and $s=\operatorname{sign}(M)$ be the signature of $M$. By Lemma 6.2 there is a manifold $M^{\prime}$ with $\beta_{2}\left(M^{\prime}\right)=m$ and $\operatorname{sign}\left(M^{\prime}\right)=s$ which supports an action of the sort in the statement of the theorem. More precisely $M^{\prime}$ is an equivariant connected sum at fixed points of standard actions on copies of $\mathbf{C} P^{2}$ and $\overline{\mathbf{C} P^{2}}$. As noted above, this action may be described as being obtained by attaching $m$ 2-handles equivariantly to the 4-ball $D^{4}$ along a framed link $K^{\prime}$ in $S^{3}$ invariant under a linear action of $\mathbf{Z}_{p}$ on $D^{4}$, and then coning off the linear action on the resulting boundary. Let $A^{\prime}$ be the linking matrix of $K^{\prime}$.

According to Lemma 2.2 there is another unimodular, symmetric $m \times m$ matrix $A$ such that $A \equiv A^{\prime}(\bmod p)$ and the intersection form of our given manifold $M$ has 
matrix $A$ with respect to a suitable basis for $H_{2}(M ; \mathbf{Z})$. As in the proof of Theorem 4.1 , we may change the framings of the components of $K^{\prime}$ by multiples of $p$, and, by equivariant isotopies of the individual components of $K^{\prime}$, change the linking numbers of pairs of components by multiples of $p$, so as to arrive at a new framed link $K$ in $S^{3}$ invariant under the same linear action on $D^{4}$ having linking matrix $A$. Note in particular that the image of each component of $K$ in the lens space $S^{3} / \mathbf{Z}_{p}$ represent the same element of $\pi_{1}\left(S^{3} / \mathbf{Z}_{p}\right)$ as the corresponding component of $K^{\prime}$ does. Moreover, the framing action associated with $K_{i}$ is the same as that associated with $K_{i}^{\prime}$.

Let $W$ be the result of equivariant 2-handle addition to $D^{4}$ along $K$; let $\Sigma=\partial W$; and let $g: W \rightarrow W$ be the generator of the $\mathbf{Z}_{p}$ action. We must check that $\alpha\left(\Sigma / \mathbf{Z}_{p}\right)$ is that of some lens space. Let $W^{\prime}, \Sigma^{\prime}$, and $g^{\prime}$ be the corresponding objects for the action on the connected sum of copies of $\mathbf{C} P^{2}$ and $\overline{\mathbf{C} P^{2}}$, yielding the lens space $\Sigma^{\prime} / \mathbf{Z}_{p}$. It follows from the $G$-Signature Theorem that $\alpha\left(\Sigma / \mathbf{Z}_{p}\right)=\alpha\left(\Sigma^{\prime} / \mathbf{Z}_{p}\right)$.

We claim that $\Delta\left(\Sigma / \mathbf{Z}_{p}\right)=\Delta\left(\Sigma^{\prime} / \mathbf{Z}_{p}\right)$ as well. This follows from Lemma 6.3, since the corresponding framing actions for the two links are the same.

Then it is a consequence of Proposition 5.3 that the action on $\Sigma$ extends locally linearly to a contractible 4-manifold $V$ with $\partial V=\Sigma$. Thus one obtains an action of the sort required by the theorem on the manifold $M^{\prime \prime}=W \cup_{\Sigma} V$.

Now $M^{\prime \prime}$ is a closed, simply connected 4-manifold with the same intersection form as $M$. If $M$ has an even intersection form, then by Freedman $[\mathbf{1 9 8 2}] M^{\prime \prime} \cong M$ and we are done. If $M$ has an odd intersection form, then $M^{\prime \prime}$ may have triangulation obstruction in $H^{4}\left(M^{\prime \prime} ; \mathbf{Z}_{2}\right) \approx \mathbf{Z}_{2}$ distinct from that of $M$. So we must see how to alter the triangulation obstruction for $M^{\prime \prime}$, just as we did in $\S 4$.

As before the link $K$ has a characteristic sublink $K_{0} \subset K$. Change $K$ to $K^{+}$by equivariantly inserting into $K_{0} p$ little trefoils with zero framing (so that $K^{+}$and $K$ have the same framing). Let $W^{+}$be the result of equivariant handle addition to $D^{4}$ along $K^{+}$, with periodic map $g^{+}$and boundary $\Sigma^{+}$. Let $M^{+}$be the closed manifold obtained by capping off $\Sigma^{+}$with a contractible manifold.

By Lemma 1.1 the framing actions on corresponding components of $K^{+}$and $K$ are the same. Therefore, consideration of $\operatorname{sign}\left(g^{+}, W^{+}\right)$shows that $\alpha\left(\Sigma^{+} / \mathbf{Z}_{p}\right)=$ $\alpha\left(\Sigma / \mathbf{Z}_{p}\right)=\alpha\left(\Sigma^{\prime} / \mathbf{Z}_{p}\right)$. It remains to determine the effect of this last alteration on Reidemeister torsion.

We claim that $\Delta\left(\Sigma^{+} / \mathbf{Z}_{p}\right)=\Delta\left(\Sigma / \mathbf{Z}_{p}\right)=\Delta\left(\Sigma^{\prime} / \mathbf{Z}_{p}\right)$, as well. Again this follows from Lemma 6.3.

7. Concluding remarks. We conclude with some observations about finding involutions on 4-manifolds with odd intersection forms and locally linear involutions on 4-manifolds with even intersection forms.

PROPOSITION 7.1. If $T$ is an orientation preserving involution on a closed, orientable 4-manifold $M$ that has an odd intersection form, and $T$ acts by the identity on $\mathrm{H}_{2}\left(M ; \mathbf{Z}_{2}\right)$, then the fixed point set $F$ of $T$ contains a 2-dimensional component.

Proof. For some $u \in H_{2}\left(M ; \mathbf{Z}_{2}\right)$ the intersection number $u \cdot u$ is nonzero $\bmod 2$. On the other hand, if $u \in$ image $\left[H_{2}\left(M-F^{2} ; \mathbf{Z}_{2}\right) \rightarrow H_{2}\left(M ; \mathbf{Z}_{2}\right)\right]$, then $u \cdot T_{*}(u) \equiv 0(\bmod 2)$. For this one can either give a direct geometric argument or 
refer to the more general spectral sequence-Steenrod squares argument in Bredon $\left[1972, \S\right.$ VII.7]. Then $u \cdot u=u \cdot T_{*}(u)=0$, a contradiction.

Recall that if $\mathbf{Z}_{p}$ acts on a manifold of the homotopy type of $\mathbf{C} P^{2}$, then the possibilities for the fixed point set are as follows: a point and a 2-sphere, three points (when $p$ is odd only), or a real projective plane (when $p=2$ only, and the action is nontrivial on $\left.H_{2}(M ; \mathbf{Z})\right)$. See Bredon [1972, §VII.3].

Proposition 7.2. Suppose that $T$ is an involution on a 4-manifold $M$ of the homotopy type of $\mathbf{C} P^{2}$, acting by the identity on $\mathrm{H}_{2}(\mathrm{M} ; \mathbf{Z})$, such that $\mathrm{T}$ is locally linear at each point of the 2-dimensional part $F^{2}$ of the fixed point set of $T$. Then $M$ is homeomorphic to $\mathbf{C} P^{2}$.

PROOF. As noted above $F^{2}$ is a 2 -sphere that represents a nontrivial $\mathbf{Z}_{2}$ homology class, and which is locally flat by the local linearity hypothesis. According to Freedman $[\mathbf{1 9 8 3}] F^{2}$ has a normal bundle in $M$. Wall's topological formulation of the $G$-Signature Theorem (Wall $[\mathbf{1 9 7 0}$, p. 188]) then applies to show that the arguments of Hsiang and Szczarba [1971] work in this situation to see that $F^{2}$ represents a generator of $\mathrm{H}_{2}(M ; \mathbf{Z}) \approx \mathbf{Z}$. It follows that the boundary of a tubular neighborhood $N$ of $F^{2}$ is the 3 -sphere, and that $\operatorname{cl}(M-N)$ is the 4 -ball, by the topological Poincaré conjecture. Thus $M$ is obtained from the Hopf 2-disk bundle over the 2 -sphere by attaching a 4 -ball. It follows that $M$ is homeomorphic to $\mathbf{C} P^{2}$ as required.

Proposition 7.2 thus shows that any involution on the fake $\mathbf{C} P^{2}$ which induces the identity on homology must have a point on its fixed 2-sphere where it is not locally linear. On the other hand, Kwasik and Vogel [1985] have constructed an involution on an odd manifold with nonvanishing triangulation obstruction and fairly large middle Betti number that is locally linear except at one isolated fixed point.

PROPOSITION 7.3. Suppose that $\mathbf{Z}_{2}$ acts homologically trivially, pseudofreely, and locally linearly on a closed, simply connected 4-manifold $M$. Then $M$ is homeomorphic to a connected sum of copies of $S^{2} \times S^{2}$, and each such manifold admits such a $\mathbf{Z}_{2}$ action.

Proof. Since the action is locally linear and pseudofree, the $G$-Signature Theorem applies to say that $\operatorname{sign}(g, M)=0$, where $g$ generates the action. Since the action is homologically trivial, $\operatorname{sign}(M)=\operatorname{sign}(g, M)=0$. By Proposition 7.1, $M$ must have an even intersection form. The intersection form is even and of signature zero. Therefore the classification theorem of Freedman applies to show that $M$ is a connected sum of copies of $S^{2} \times S^{2}$. One easily constructs the desired examples of involutions on the standard building blocks. Since the building blocks admit orientation reversing homeomorphisms, one may form equivariant connected sums at fixed points to construct the required actions.

Finally we denote the analogue for $p=2$ of the Locally Linear Extension Theorem, Proposition 5.3.

PROPOSITION 7.4. A free $\mathbf{Z}_{2}$ action on a homology 3-sphere $\Sigma$ extends to a locally linear action on a contractible 4-manifold if and only if $\alpha\left(\Sigma / \mathbf{Z}_{2}\right)=0$.

Proof SKETCH. The necessity of the $\alpha$-invariant condition is just as before. By Lemma 3.2 there is a degree one normal map $f: \Sigma / \mathbf{Z}_{2} \rightarrow \mathbf{R} P^{3}$. By Taylor 
[1984, Lemma 2.1], $f$ is normally cobordant to the identity $\mathbf{R} P^{3} \rightarrow \mathbf{R} P^{3}$. The normal bordism yields a 4-dimensional surgery problem mapping to $\mathbf{R} P^{3} \times I$ that is a simple $\mathbf{Z}\left[\mathbf{Z}_{2}\right]$ homology equivalence over the ends. The surgery obstruction is then completely captured by the $\alpha$-invariant.

\section{REFERENCES}

G. E. Bredon, Introduction to compact transformation groups, Academic Press, New York 1972.

S. E. Cappell and J. L. Shaneson, The codimension two placement problem and homology equivalent manifolds, Ann. of Math. (2) 99 (1974), 277-348.

A. L. Edmonds, Preliminary notes on finite group actions on four-manifolds, unpublished notes, 1984.

M. H. Freedman, The topology of four-dimensional manifolds, J. Differential Geom. 17 (1982), 357-453.

_ Poland, 1983, pp. 647-663.

M. J. Greenberg and J. R. Harper, Algebraic topology: a first course. Benjamin/Cummings, Reading, Mass., 1981.

F. Hirzebruch, W. D. Neumann, and S. S. Koh, Differentiable manifolds and quadratic forms, Marcel Dekker, New York, 1971.

F. Hirzebruch and D. Zagier, The Atiyah-Singer index theorem and elementary number theory, Publish or Perish, Boston, 1973.

W.-C. Hsiang and R. H. Szczarba, On embedding surfaces in four-manifolds, Proc. Sympos. Pure Math., vol. 22, Amer. Math. Soc., Providence, R.I., 1971, pp. 97-103.

S. Kwasik, On symmetries of the fake $C P^{2}$, Preprint, 1985.

S. Kwasik and P. Vogel, Asymmetric four-dimensional manifolds, Preprint, 1984. Non-locally smoothable topological symmetries of four manifolds, Preprint, 1985.

J. W. Milnor, A duality theorem for Reidemeister torsion, Ann. of Math. (2) 76 (1962), 137-147. , Whitehead torsion, Bull. Amer. Math. Soc. 72 (1966), 358-426.

L. R. Taylor, Relative Rochlin invariant, Topology Appl. 18 (1984), 259-280.

C. T. C. Wall, Surgery on compact manifolds, Academic Press, London, 1970.

S. H. Weintraub, Semi-free $\mathbf{Z}_{p}$-actions on highly-connected manifolds, Math. Z. 145 (1975), 163-185.

, Topological realization of equivariant intersection forms, Pacific J. Math. 73 (1977), 257-280.

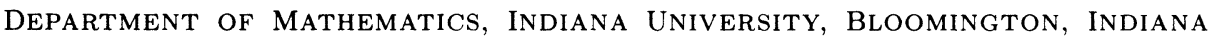
47405 